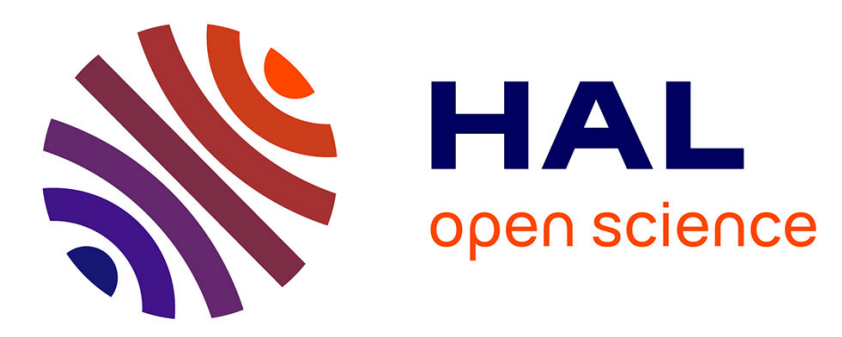

\title{
A mathematical modeling approach for high and new technology-project portfolio selection under uncertain environments
}

\author{
Vahid Mohagheghi, S. Meysam Mousavi, Behnam Vahdani, Ali Siadat
}

\section{- To cite this version:}

Vahid Mohagheghi, S. Meysam Mousavi, Behnam Vahdani, Ali Siadat. A mathematical modeling approach for high and new technology-project portfolio selection under uncertain environments. Journal of Intelligent and Fuzzy Systems, 2017, 32 (6), pp.4069-4079. 10.3233/jifs-152510 . hal-02436512

\section{HAL Id: hal-02436512 \\ https://hal.science/hal-02436512}

Submitted on 13 Jan 2020

HAL is a multi-disciplinary open access archive for the deposit and dissemination of scientific research documents, whether they are published or not. The documents may come from teaching and research institutions in France or abroad, or from public or private research centers.
L'archive ouverte pluridisciplinaire HAL, est destinée au dépôt et à la diffusion de documents scientifiques de niveau recherche, publiés ou non, émanant des établissements d'enseignement et de recherche français ou étrangers, des laboratoires publics ou privés. 


\title{
A mathematical modeling approach
}

\section{portfolio selection under uncertain environments}

\author{
Vahid Mohagheghi ${ }^{\mathrm{a}}$, S. Meysam Mousavi ${ }^{\mathrm{a}, *}$, Behnam Vahdani $^{\mathrm{b}}$ and Ali Siadat ${ }^{\mathrm{c}}$ \\ ${ }^{a}$ Department of Industrial Engineering, Faculty of Engineering, Shahed University, Tehran, Iran \\ ${ }^{\mathrm{b}}$ Faculty of Industrial and Mechanical Engineering, Qazvin Branch, Islamic Azad University, Qazvin, Iran \\ ${ }^{\mathrm{c}}$ LCFC, Arts et Métier Paris Tech, Centre de Metz, Metz, France
}

\begin{abstract}
High and new technology-project as a tool to achieve productive forces through scientific and technological knowledge is characterized as knowledge based with high risk and returns. Often conflicting objectives of these projects have complicated their assessment and selection process. This paper offers a novel approach of high technology-project portfolio selection in two main parts. In the first part, a new risk reduction compromise decision-making model is proposed that applies a new approach in determining the weights of experts and in avoiding information loss. The objective function of a new interval type-2 fuzzy sets (IT2Fs) based mathematical model of project portfolio selection is formed by the outcome. To depict model's applicability, data from case study of high technology-project selection in the literature is used to present the efficacy of the model.
\end{abstract}

Keywords: High and new technology-projects, project portfolio selection, compromise solutions, mathematical modeling, interval type-2 fuzzy sets

\section{Introduction}

Large high-tech mega-projects are referred to projects that require research and development and/or application of technology in addition to a substantial infrastructure and multi-million or even billion dollar budgets. Additionally, their time-horizons are measured in at least years [18]. Ability of decision makers (DMs)' to flawlessly analyze projects is weakened by high risk of uncertainty or inadequacy of project data

*Corresponding author. S. Meysam Mousavi, Department of Industrial Engineering, Faculty of Engineering, Shahed University, Tehran, Iran. Tel.: +98 21 51212091; E-mail: sm.mousavi@shahed.ac.ir.
$[11,21-24,31]$. This complication and vagueness is intensified in high-technology [16].

High-tech mega-projects have high levels of risk, vagueness and uncertainty. At the initial phases, uncertainty mostly affects performance expectations, political environments, goals, motivations, and potentials [25, 11]. Thomas and Mengel [10] stated that complex projects have vagueness and ambiguity of the not-yet-known that occur as events that crucially reframe meaning, interpretation, and social significance emerge.

Due to lack of adequate historical data, vagueness and high influence of experts' judgments on project selection problems, fuzzy sets theory has been referred to as a welcomed approach in considering 
project uncertainty $[3,27]$. Most of the studies even in the recent years are based on classic fuzzy sets. Zadeh [13] expanded type-2 fuzzy sets (FSs). Type2 FSs have fuzzy membership functions (MFs) also called "membership of membership". In type-2 FSs in contrast to type-1 FSs each membership value of each element is expressed by fuzzy set in $[0,1]$, rather than using a crisp number in $[0,1]$. Despite all these positive points, unfortunately using T2FSs to model the environment of high-tech project is still new.

Some of the main literature gaps that motivated proposing this paper are as follows: (1) literature of project selection and projects portfolio selection is very weak when it comes to high-tech projects (2) this problem contains very high levels of uncertainty and vagueness and they are not yet well addressed; (3) the existing decision-making methods do not comprehensively address risk of uncertainty and importance of each DM's judgment.

In order to fill the gaps of this practical decisionmaking situation, this paper offers a novel two-part model of high-tech project portfolio selection under highly uncertain and vague conditions is proposed that presents interval type-2 fuzzy sets (IT2FSs) to model uncertainty. In the first part of the presented approach, a new IT2FSs based-risk reduction compromise decision-making process is presented that avoids information loss in designating weights to DMs. Employing IT2FSs gives the model with high power of uncertainty modeling and calculating. Moreover, each DM receives a weight based on the judgments received in the process. In the second part, a new mathematical model of project portfolio selection with IT2F-constraints is proposed to find the optimal portfolio of projects. Eventually, in this paper the basic concept of IT2FSs is improved by presenting a novel method of interval type-2 fuzzy number (IT2FN)-ranking.

The following illustrates the remainder of this paper. In Section 2, the relevant literature on compromise decision making problems is reviewed. Section 3 displays the introduced model. Model's application is illustrated in Section 4 and eventually Section 5 presents the conclusion remarks.

\section{Literature review}

Most of the project selection related studies apply the concept of multi-criteria decision-making and multi-criteria analysis [1]. Actually, since project evaluation and selection is a group decision-making process that is affected by different project aspects, applying multi criteria decision-making methods could be a useful approach. On the other hand, one aspect that highly influences project evaluation and selection studies especially in case of high technology-projects is uncertainty. Over the years, a large number of fuzzy multi-criteria decision making (FMCDM) methods have been introduced. All approaches are mainly concerned with conducting the decision-making process better informed and more structured. Through reviewing previous studies, FMCDM can be categorized as a fuzzy multi-objective decision-making (FMODM) and fuzzy multi-attribute decision-making (FMADM) approach.

A practical solution in highly uncertain environments is applying type-2 FSs. The development made by $\mathrm{Wu}$ and Mendel [2] was based on using words for interval type-2 fuzzy hierarchical MADM. The model was applied to assess a weapon system. Dereli and Alton [26] used IT2FSs to present a framework that evaluated technologies. Dereli and Alton [25] further investigated the problem of candidate technology assessment with the help of a fuzzy inference system that used type 2 fuzzy sets. Qin et al. [9] developed a decision model integrating VIKOR method and prospect theory. To illustrate the applicability of their method, they used case study of a high-tech risk evaluation.

Another approach in using IT2FSs in project environment is employing these sets in mathematical modeling and programming. To the best of our knowledge, this approach in project and project portfolio selection is new and only a small number of studies have used this approach. For instance, Mohagheghi et al. [28] presented a model of project cash flow prediction that could also be applied in project evaluation and appraisal. Mohagheghi et al. [29] applied IT2FSs to evaluate $R \& D$ project evaluation and project portfolio selection. As mentioned earlier employing type-2 FSs in mathematical modeling for project selection problems is new and most of the IT2FSbased approaches apply different MCDM techniques.

Since this paper offers a new method of IT2Franking, a brief review of ranking methods is presented. Mitchell [4] presented one of the first type2 fuzzy-ranking methods. The method was based on random inputs and the randomness involved in the process would affect the final results. Qin and Liu [9] used operators of arithmetic average, geometric average and harmonic average (HA) to rank IT2FNs. Kunda et al. [19] presented a model of interval type-2 
fuzzy-ranking. The method was based on the concept of using relative preference index. Proposed ranking approaches are not totally satisfactory. Some of the reasons are as follows: lack of enough discrimination while differentiating similar IT2FNs, having inconsistent and sometimes counter-intuitive results under different situations, and requiring large computational effort under specific conditions.

As it was mentioned, any practical project evaluation process requires sophisticated consideration of uncertainty. Most of the existing literature of the project and project portfolio selection is based on classic fuzzy sets theory. In environments like high technology-project environments that have a very high level of uncertainty it is more practical to use type-2 FSs. Therefore, in this paper, a new model of project portfolio selection under an IT2Fenvironment that controls the risk of uncertainty in addition to avoiding information loss when giving weight to DMs is proposed.

\section{Proposed approach}

In this section, first a new effective ranking method is presented that is based on the concept of positive and negative ideal solutions. The project portfolio selection has two main parts. In the first part, a novel decision-making approach is presented that avoids information loss in addition to controlling uncertainty of soft computing. This part of the model results in ranking the candidate projects while considering the selection criteria. The second part includes a new mathematical model based on the concept of IT2FSs that uses the results of the previous part of the model to select the best portfolio of projects while considering conflicting and practical limitations and considerations. It should be noted that the applied IT2FS definitions and operators were taken from $[6-8,12,15,20]$.

\subsection{Proposed ranking trapezoidal interval type-2 fuzzy numbers}

In this section, a novel approach for comparing and ranking IT2FNs is presented. This approach is based on sensible use of concept of ideal solutions. Also, a distance-based similarity measure between IT2FNs is appropriately developed for effectively obtaining the overall performance for any given IT2FN ranking and comparing process. This method is based on the studies of Deng [5], Ren et al. [14], Mohagheghi et al.
[30] and Zhang and Zhang [34]. The step-by-step algorithm is introduced as follows:

1. Define the trapezoidal interval type- 2 fuzzy positive ideal solution as $\tilde{\tilde{X}}_{\max }$ and the negative ideal solution as $\tilde{\tilde{X}}_{\text {min }}$.

2. Calculate the distance-based degree of similarity between each interval type- 2 fuzzy number $\tilde{\tilde{A}}_{i}(i=1,2, \ldots, n)$ and the positive interval type-2 fuzzy ideal solution $\left(d_{i}^{+}\right)$by applying Equation (1):

$$
\begin{aligned}
& d_{i}^{+}\left(\tilde{\tilde{A}}_{i}, \tilde{\tilde{X}}_{\max }\right) \\
& =\sqrt{\begin{array}{c}
\sum_{i=1}^{4}\left(a_{i}^{U}-x_{i}^{U}\right)^{2}+\sum_{i=1}^{4}\left(a_{i}^{L}-x_{i}^{L}\right)^{2} \\
+\sum_{i=1}^{2}\left(H_{i}\left(\tilde{\tilde{A}}^{U}\right)-H_{i}\left(\tilde{\tilde{X}}^{U}\right)\right)^{2} \\
+\sum_{i=1}^{2}\left(H_{i}\left(\tilde{\tilde{A}}^{L}\right)-H_{i}\left(\tilde{\tilde{X}}^{L}\right)\right)^{2}
\end{array}}
\end{aligned}
$$

3. Calculate the distance-based degree of similarity between each interval type- 2 fuzzy number $\tilde{\tilde{A}}_{i}(i=1,2, \ldots, n)$ and the negative interval type-2 fuzzy ideal solution $\left(d_{i}^{-}\right)$by applying Equation (2):

$$
\begin{aligned}
& d_{i}^{-}\left(\tilde{\tilde{A}}_{i}, \tilde{\tilde{X}}_{\text {min }}\right) \\
& =\sqrt{\begin{array}{c}
\sum_{i=1}^{4}\left(a_{i}^{U}-x_{i}^{U}\right)^{2}+\sum_{i=1}^{4}\left(a_{i}^{L}-x_{i}^{L}\right)^{2} \\
+\sum_{i=1}^{2}\left(H_{i}\left(\tilde{\tilde{A}}^{U}\right)-H_{i}\left(\tilde{\tilde{X}}^{U}\right)\right)^{2} \\
+\sum_{i=1}^{2}\left(H_{i}\left(\tilde{\tilde{X}}^{L}\right)-H_{i}\left(\tilde{X}^{L}\right)\right)^{2}
\end{array}}
\end{aligned}
$$

4. Determine the point $E\left(\min \left(d_{i}^{+}\right), \max \left(d_{i}^{-}\right)\right)$, which is referred to as the optimized ideal reference point.

5. Calculate the distance from each alternative to point $E$ by using the following:

$$
\begin{gathered}
E D_{i}=\sqrt{\begin{array}{l}
{\left[d_{i}^{+}-\min \left(d_{i}^{+}\right)\right]^{2}} \\
+\left[d_{i}^{-}-\max d_{i}^{-}\right]^{2},
\end{array}} \\
i=1,2, \ldots, n
\end{gathered}
$$

6. Rank the interval type-2 fuzzy numbers $\tilde{\tilde{A}}_{i}(i=1,2, \ldots, n)$ in increasing order of $E D_{i}$. If two numbers happen to have the same value 
Table 1

Linguistic terms and trapezoidal interval type-2 fuzzy numbers

\begin{tabular}{ll}
\hline Linguistic variables & Trapezoidal interval type-2 fuzzy numbers \\
\hline Extreme High (EH) & $((8,9,9,10 ; 1,1),(8.5,9,9,9.5 ; 0.9,0.9))$ \\
Very High (VH) & $((6,7,7,8 ; 1,1),(6.5,7,7,7.5 ; 0.9,0.9))$ \\
High $(\mathrm{H})$ & $((4,5,5,6 ; 1,1),(4.5,5,5,5.5 ; 0.9,0.9))$ \\
Medium High (MH) & $((2,3,3,4 ; 1,1),(2.5,3,3,4.5 ; 0.9,0.9))$ \\
M (Medium) & $((1,1,1,1 ; 1,1),(1,1,1,1 ; 0.9,0.9))$ \\
Medium Low (ML) & $((0.25,0.33,0.33,0.5 ; 1,1),(0.22,0.33,0.33,0.4 ; 0.9,0.9))$ \\
Low (L) & $((0.17,0.2,0.2,0.25 ; 1,1),(0.18,0.2,0.2,0.22 ; 0.9,0.9))$ \\
Very Low (VL) & $((0.13,0.14,0.14,0.17 ; 1,1),(0.13,0.14,0.14,0.15 ; 0.9,0.9))$ \\
Extreme Low (EL) & $((0.1,0.11,0.11,0.13 ; 1,1),(0.11,0.11,0.11,0.12 ; 0.9,0.9))$ \\
\hline
\end{tabular}

of $E D_{i}$, determine their $E D_{i}$ by the following Equation and rank them in increasing order of $E D_{i}$.

$$
E D_{i}=d_{i}^{+}-\min \left(d_{i}^{+}\right) .
$$

\subsection{Proposed type 2-risk reduction compromise ratio model}

In this section, a new risk reduction compromise ratio method based on trapezoidal IT2FSs and footthe impacts of the criteria used in the decisionmaking process. Linguistic variables were converted into trapezoidal interval type- 2 fuzzy sets and are presented in Table 1. This novel method can be described in detail by means of the following.

First, decision information of each DM should be gathered, therefore:

$$
\tilde{\tilde{D}}_{K}=\left(\tilde{\tilde{D}}_{i j}^{K}\right)_{m \times n}=\left[\begin{array}{ccc}
\tilde{D}_{11}^{K} & \cdots & \tilde{\tilde{D}}_{1 n}^{K} \\
\vdots & \ddots & \vdots \\
\tilde{\tilde{D}}_{m 1}^{K} & \cdots & \tilde{\tilde{D}}_{m n}^{K}
\end{array}\right]
$$

$$
\tilde{\tilde{W}}_{K}=\left(\tilde{\tilde{w}}_{1}^{k}, \tilde{\tilde{w}}_{2}^{k}, \ldots, \tilde{\tilde{w}}_{n}^{k}\right), K \in T
$$

Where $\tilde{\tilde{D}}_{K}$ is the decision matrix and $\tilde{\tilde{W}}_{K}$ is the weight vector of attributes, $m$ is the number of criteria, $n$ is the number of alternatives compared and $T$ denotes the group of experts. $\tilde{\tilde{w}}_{j}$ is the weight vector of the criteria. Obviously, $\tilde{\tilde{D}}_{i j}^{K}$ and $\tilde{\tilde{W}}_{K}$ are trapezoidal IT2FSs.

The decision matrix should be normalized $(\tilde{\tilde{F}})$ using Equations (8 and 9).

$$
\tilde{\tilde{F}}=\left[\begin{array}{ccc}
\tilde{F}_{11} & \cdots & \tilde{F}_{1 n} \\
\vdots & \ddots & \vdots \\
\tilde{F}_{m 1} & \cdots & \tilde{F}_{m n}
\end{array}\right]
$$
print of uncertainty (FOU) is developed that explores

$$
\begin{aligned}
\tilde{F}_{i j}= & \left(f_{i 1}^{U}, f_{i 2}^{U}, f_{i 3}^{U}, f_{i 4}^{U} ;\left(\min H_{i 1}\left(D_{1}^{U}\right), H_{1}\left(d^{*}\right)\right)\right. \\
& \left(\min H_{2}\left(D_{1}^{U}\right), H_{2}\left(d^{*}\right)\right), f_{i 1}^{L}, f_{i 2}^{L}, f_{i 3}^{L}, f_{i 4}^{L} ; \\
& \left(\min H_{1}\left(D_{1}^{L}\right), H_{1}\left(d^{*}\right)\right),\left(\min H_{2}\left(D_{1}^{L}\right),\right. \\
& \left.\left.H_{2}\left(d^{*}\right)\right)\right)
\end{aligned}
$$

where

$$
f_{1 i}^{T}
$$$$
=\min \left(\frac{d_{i j_{1 m}}^{T}}{d^{*}}, \frac{d_{i j_{1 m}}^{T}}{d^{*}}, \frac{d_{i j_{1(5-m)}}^{T}}{d^{*}}, \frac{d_{i j_{1(5-m)}}^{T}}{d^{*}}\right), \quad{ }_{242}
$$$$
T \in\{U, L\}, m \in\{1,2\}
$$

$\tilde{F}_{i j}=\left(f_{i 1}^{U}, f_{i 2}^{U}, f_{i 3}^{U}, f_{i 4}^{U} ;\left(\min H_{i 1}\left(D_{1}^{U}\right), H_{1}\left(d^{-}\right)\right)\right.$, $\left(\min H_{2}\left(D_{1}^{U}\right), H_{2}\left(d^{-}\right)\right), f_{i 1}^{L}, f_{i 2}^{L}, f_{i 3}^{L}, f_{i 4}^{L} ; \quad 250$ $\left(\min H_{1}\left(D_{1}^{L}\right), H_{1}\left(d^{-}\right)\right),\left(\min H_{2}\left(D_{1}^{L}\right)\right.$,

$$
\left.\left.H_{2}\left(d^{-}\right)\right)\right)
$$

where

$$
\begin{aligned}
& f_{1 i}^{T}=\min \left(\frac{d^{-}}{d_{i j_{2 m}}^{T}}, \frac{d^{-}}{d_{i j_{2(5-m)}}^{T}}, \frac{d^{-}}{d_{i j_{2 m}}^{T}}, \frac{d^{-}}{d_{i j_{2(5-m)}}^{T}}\right), \\
& f_{1 j}^{T}=\min \left(\frac{d^{-}}{d_{i j_{2(5-n)}}^{T}}, \frac{d^{-}}{d_{i j_{2 n}}^{T}}, \frac{d^{-}}{d_{i j_{2(5-n)}}^{T}}, \frac{d^{-}}{d_{i j_{2 n}}^{T}}\right), \\
& T \in\{U, L\}, n \in\{3,4\} \\
& i=1,2, \ldots, n, j \in B
\end{aligned}
$$


Where $B$ denotes the group of benefit criteria and $C$ represents the group of cost criteria. $d^{*}$ and $d^{-}$are also obtained as follows:

$$
\begin{aligned}
& d^{*}=\max _{i}\left(d_{i j}\right)_{4}^{U} \\
& d^{-}=\min _{i}\left(d_{i j}\right)_{1}^{U}
\end{aligned}
$$

The normalized weighted decision matrix is calculated by employing Equation (12).

$$
\begin{aligned}
\tilde{\tilde{G}}= & {\left[\begin{array}{ccc}
\tilde{\tilde{G}}_{11} & \cdots & \tilde{\tilde{G}}_{1 n} \\
\vdots & \ddots & \vdots \\
\tilde{\tilde{G}}_{m 1} & \cdots & \tilde{\tilde{G}}_{m n}
\end{array}\right] } \\
\tilde{\tilde{G}}_{i j}= & \tilde{\tilde{F}}_{i j} \times \tilde{\tilde{w}}_{j} \\
= & \left(g_{i 1}^{U}, g_{i 2}^{U}, g_{i 3}^{U}, g_{i 4}^{U} ;\left(\min H_{i 1}\left(G_{1}^{U}\right), H_{1}\left(F_{1}^{U}\right)\right),\right. \\
& \left(\min H_{i 1}\left(G_{1}^{U}\right), H_{1}\left(F_{1}^{U}\right)\right), \\
& g_{i 1}^{L}, g_{i 2}^{L}, g_{i 3}^{L}, g_{i 4}^{L} ;\left(\min H_{1}\left(G_{1}^{L}\right), H_{1}\left(F_{1}^{L}\right)\right), \\
& \left(\min H_{2}\left(G_{1}^{L}\right), H_{2}\left(F_{1}^{L}\right)\right)
\end{aligned}
$$

where

$$
g_{1 i}^{T}=\min \left(\begin{array}{c}
f_{i j_{1 m}}^{T} w_{j_{2 m}}^{T}, f_{i j_{1 m}}^{T} w_{j_{2(5-m)}}^{T}, \\
f_{i j_{1(5-m)}}^{T} w_{j_{2 m}}^{T}, f_{i j_{1(5-m)}}^{T} w_{j_{2(5-m)}}^{T}
\end{array}\right),
$$

$$
T \in\{U, L\}, m \varepsilon\{1,2\}
$$

$$
\begin{gathered}
g_{1 j}^{T}=\min \left(\begin{array}{c}
f_{i j_{1(5-n)}}^{T} w_{j_{2(5-n)}}^{T}, \\
f_{i j_{1(5-n)}}^{T} w_{j_{2 n}}^{T}, f_{i j_{1 n}}^{T} w_{j_{2(5-n)}}^{T}, f_{i j_{1 n}}^{T} w_{j_{2 n}}^{T}
\end{array}\right), \\
T \in\{U, L\}, n \varepsilon\{3,4\}
\end{gathered}
$$

The ideal decisions of all individual decisions in mean sense should be the average of all individual decisions. A negative ideal decision should be of the maximum separation from the positive ideal decision [32]. Therefore, the best decision $\left(G^{*}\right)$, the left negative ideal decision $\left(G_{l}^{-}\right)$and the right negative ideal decision $\left(G_{r}^{-}\right)$are calculated by applying the following equations:

$$
\tilde{\tilde{G}}^{*}=\left[\begin{array}{ccl}
\tilde{\tilde{g}}_{11}^{*} & \cdots & \tilde{\tilde{g}}_{1 n}^{*} \\
\vdots & \ddots & \vdots \\
\tilde{\tilde{g}}_{m 1}^{*} & \cdots & \tilde{\tilde{g}}_{m n}^{*}
\end{array}\right]
$$

$$
\begin{aligned}
\tilde{\tilde{g}}_{i j}^{*}= & \left(1 / t \sum_{k=1}^{t} g_{i j 1}^{U}, 1 / t \sum_{k=1}^{t} g_{i j 2}^{U}, 1 / t \sum_{k=1}^{t} g_{i j 3}^{U}\right. \\
& 1 / t \sum_{k=1}^{t} g_{i j 4}^{U} ;\left(H_{i 1}\left(G_{1}^{U}\right)\right),\left(H_{i 2}\left(G_{1}^{U}\right)\right), \\
& 1 / t \sum_{k=1}^{t} g_{i j 1}^{L}, 1 / t \sum_{k=1}^{t} g_{i j 2}^{L}, 1 / t \sum_{k=1}^{t} g_{i j 3}^{L}, \\
& 1 / t \sum_{k=1}^{t} g_{i j 4}^{L} ;\left(\min H_{1}\left(G_{1}^{L}\right), H_{1}\left(F_{1}^{L}\right)\right), \\
& \left.\left(\min H_{2}\left(G_{1}^{L}\right), H_{2}\left(F_{1}^{L}\right)\right)\right)
\end{aligned}
$$

$$
\tilde{\tilde{G}}_{L}^{-}=\left[\begin{array}{ccc}
\tilde{\tilde{g}}_{l_{11}} & \ldots & \tilde{\tilde{g}}_{l_{1 n}}- \\
\vdots & \ddots & \vdots \\
\tilde{\tilde{g}}_{l_{m 1}}- & \ldots & \tilde{\tilde{g}}_{l_{m n}}-
\end{array}\right]
$$

where $\tilde{\tilde{g}}_{l_{i j}}^{-}=\min _{1 \leq k \leq t}\left\{\tilde{\tilde{g}}_{i j}^{k}\right\}$

$$
\tilde{\tilde{G}}_{R}^{-}=\left[\begin{array}{ccc}
\tilde{\tilde{g}}_{r_{11}}- & \ldots & \tilde{\tilde{g}}_{r_{1 n}}- \\
\vdots & \ddots & \vdots \\
\tilde{\tilde{g}}_{r_{m 1}}- & \ldots & \tilde{\tilde{g}}_{r_{m n}}-
\end{array}\right]
$$

where $\tilde{\tilde{g}}_{r_{i j}}^{-}=\max _{1 \leq k \leq t}\left\{\tilde{\tilde{g}}_{i j}^{k}\right\}$

The difference of each individual judgment from the ideal judgments including positive ideal decision, the left negative ideal decision and the right negative ideal decision are respectively denoted by $d_{k}^{*}, d_{l}^{-}$and $d_{r}^{-}$and are determined by the following Equations:

$$
d_{l}^{*}=\sqrt{\begin{array}{l}
\sum_{p=1}^{4}\left(g_{i j p}^{U}{ }^{k}-g_{i j p}^{U}{ }^{*}\right)^{2}+\sum_{p=1}^{4}\left(g_{i j p}^{L}{ }^{k}-g_{i j p}^{L}{ }^{*}\right)^{2} \\
+\sum_{p=1}^{2}\left(H_{p}\left(\tilde{\tilde{G}}_{l}^{U^{K}}\right)-H_{p}\left(\tilde{\tilde{G}}_{l}^{U *}\right)\right)^{2} \\
+\sum_{p=1}^{2}\left(H_{p}\left(\tilde{\tilde{G}}_{l}^{L^{K}}\right)-H_{p}\left(\tilde{\tilde{G}}_{l}^{L *}\right)\right)^{2}
\end{array}}
$$




$$
\begin{aligned}
& \sum_{p=1}^{4}\left(g_{i j p}^{U}{ }^{k}-g_{i j p}^{U}{ }^{l}\right)^{2}+\sum_{p=1}^{4}\left(g_{i j p}^{L}{ }^{k}-g_{i j p}^{L}{ }^{l}\right)^{2} \\
& d_{l}^{-}=\quad+\sum_{p=1}^{2}\left(H_{p}\left(\tilde{\tilde{G}}_{l}^{U^{K}}\right)-H_{p}\left(\tilde{\tilde{G}}_{l}^{U^{l}}\right)\right)^{2} \\
& \sqrt{+\sum_{p=1}^{2}\left(H_{p}\left(\tilde{\tilde{G}}_{l}^{L^{K}}\right)-H_{p}\left(\tilde{\tilde{G}}_{l}^{L^{l}}\right)\right)^{2}} \\
& \sum_{p=1}^{4}\left(g_{i j p}^{U}{ }^{k}-g_{i j p}^{U}{ }^{r}\right)^{2}+\sum_{p=1}^{4}\left(g_{i j p}^{L}{ }^{k}-g_{i j p}^{L}{ }^{l}\right)^{2} \\
& d_{r}^{-}=+\sum_{p=1}^{2}\left(H_{p}\left(\tilde{\tilde{G}}_{l}^{U^{K}}\right)-H_{p}\left(\tilde{\tilde{G}}_{l}^{U^{r}}\right)\right)^{2}+ \\
& \sqrt{\sum_{p=1}^{2}\left(H_{p}\left(\tilde{\tilde{G}}_{l}^{L^{K}}\right)-H_{p}\left(\tilde{\tilde{G}}_{l}^{L^{r}}\right)\right)^{2}}
\end{aligned}
$$

The closeness coefficient of the individual decision $\left(R_{k}\right)$ with respect to ideal decisions denoted by $\left(C C_{k}\right)$ is achieved as follows:

$$
C C_{k}=\frac{d_{l}^{r}+d_{l}^{l}}{d_{l}^{l}+d_{l}^{r}+d_{l}^{*}}, K \in T
$$

It is considered that larger value of $C C_{k}$ determines more importance on $k$ th DM opinion, and bigger value of weight for $k$ th DM [33]. The importance of an expert in his/her area of expertise is referred to as the individual importance and denoted by $I M_{k}$. Combination of the two DM importance considerations can be obtained as follows:

$$
\pi_{k}=\alpha I M_{k}+(1-\alpha) C C_{k}, K \in T
$$

where $\alpha(0 \leq \alpha \leq 1)$ is the optimistic coefficient that indicates whose value can be chosen according to group's opinions, $I M_{k}\left(0 \leq I M_{k} \leq 1\right)$ is the measure of importance of $k$ th DM as an expert in his/her own area of expertise.

Eventually, the weights of DMs are obtained as follows:

$$
\mu_{k}=\frac{\pi_{k}}{\sum_{k=1}^{t} \pi_{k}}, K \in T
$$

The weighted (on attributes and DMs) decision matrix $(S)$ for each DM is calculated by the following:

$$
\begin{aligned}
\tilde{\tilde{S}}_{k} & =\left(s_{i j}\right)_{m \times n}=\left(\mu_{k} \times g_{i j}^{k}\right)_{m \times n} \\
& =\left[\begin{array}{ccc}
\widetilde{s}_{11}^{k} & \cdots & \widetilde{\tilde{s}}_{1 n}^{k} \\
\vdots & \ddots & \vdots \\
\widetilde{s}_{m 1}^{k} & \cdots & \widetilde{s}_{m n}^{k}
\end{array}\right]
\end{aligned}
$$

where

$$
\begin{aligned}
\tilde{\tilde{s}}_{i j}= & \left(\mu_{k} g_{i j 1}^{U}, \mu_{k} g_{i j 2}^{U}, \mu_{k} g_{i j 3}^{U}, \mu_{k} g_{i j 4}^{U} ;\right. \\
& \left(H_{i 1}\left(G_{1}^{U}\right), H_{i 2}\left(G_{1}^{U}\right)\right), \mu_{k} g_{i j 1}^{L}, \mu_{k} g_{i j 2}^{L}, \\
& \mu_{k} g_{i j 3}^{L}, \mu_{k} g_{i j 4}^{L} ;\left(H_{i 1}\left(G_{1}^{L}\right), H_{i 2}\left(G_{1}^{L}\right)\right)
\end{aligned}
$$

The individual decision, which is weighted on attributes and DMs, is converted into the group decision, for each alternative. This is done by the following Equation:

$$
\widetilde{\tilde{S}}_{i}=\left(s_{k j}\right)_{j \times n}=\left[\begin{array}{ccc}
\widetilde{s}_{11}^{i} & \cdots & \tilde{\tilde{s}}_{1 n}^{i} \\
\vdots & \ddots & \vdots \\
\widetilde{s}_{t 1}^{i} & \cdots & \widetilde{\tilde{s}}_{t n}^{i}
\end{array}\right], i \in M,
$$

To manage the risk of uncertainty in the process the following mathematical model for each alternative is presented.

$H_{i}=\max \left(\sum_{i=1}^{M} \tilde{q}_{B i}-\sum_{i=1}^{M} \tilde{q}_{C i}\right)$

Subject to :

$\tilde{q}_{B i}=\sum_{j \in B} \sqrt{\frac{1}{4}\left(\begin{array}{c}\left(\left(s_{i j}\right)_{1}\right)^{2}+\left(\left(s_{i j}\right)_{2}\right)^{2}+\left(\left(s_{i j}\right)_{3}\right)^{2} \\ +\left(\left(s_{i j}\right)_{4}\right)^{2}\end{array}\right)}$

$\tilde{q}_{C i}=\sum_{j \in C} \sqrt{\frac{1}{4}\left(\begin{array}{l}\left(\left(s_{i j}\right)_{1}\right)^{2}+\left(\left(s_{i j}\right)_{2}\right)^{2}+ \\ \left(\left(s_{i j}\right)_{3}\right)^{2}+\left(\left(s_{i j}\right)_{4}\right)^{2}\end{array}\right)}$

$\tilde{S}_{i j}=\left(s_{i j_{1}}, s_{i j_{2}}, s_{i j_{3}}, s_{i j_{4}}\right)$

$s_{i j_{1}}^{U} \leq s_{i j_{1}} \leq s_{i j_{1}}^{L} j=1, \ldots, m, i=1, \ldots, n$

$s_{i j_{2}}^{U} \leq s_{i j_{2}} \leq s_{i j_{2}}^{L} j=1, \ldots, m, i=1, \ldots, n$

$s_{i j_{3}}^{L} \leq s_{i j_{3}} \leq s_{i j_{3}}^{U} j=1, \ldots, m, i=1, \ldots, n$

$s_{i j_{4}}^{L} \leq s_{i j_{4}} \leq s_{i j_{4}}^{U} j=1, \ldots, m, \quad i=1, \ldots, n$
311 
$Q U_{i}$, Score of project $i$ obtained in Section 3.2, below:

$$
Q U_{i}=\left[\frac{H_{i}}{H_{\max }}\right] \times 100 \%
$$
its benefit and cost criteria.

\subsection{Proposed mathematical model} follows:

IT2F investment project $i$, ment,

$$
\left(\begin{array}{c}
I T 2 F H R i_{1}^{U}, I T 2 F H R i_{2}^{U}, I T 2 F H R i_{3}^{U}, I T 2 F H R i_{4}^{U} ; \\
H_{1}\left(I T 2 F H R i_{1}^{U}\right), H_{2}\left(I T 2 F H R i_{1}^{U}\right)
\end{array}\right),
$$

$$
\left(\begin{array}{c}
I T 2 F H R i_{1}^{L}, I T 2 F H R i_{2}^{L}, I T 2 F H R i_{3}^{L}, I T 2 F H R i_{4}^{L} ; \\
H_{1}\left(I T 2 F H R i_{1}^{L}\right), H_{2}\left(I T 2 F H R i_{1}^{L}\right)
\end{array}\right),
$$

Where $\varepsilon$ denotes the maximum amount of acceptable uncertainty. This amount is imposed on the mathematical problem by Equation (33). In this step based on the concept of FOU, the IT2FNs are converted to type- 1 fuzzy sets. These new fuzzy numbers are made in the limits of the initial IT2FNs by Equations (29-32). The area between the lower and upper limits of an IT2FS is known as FOU. The presented approach aims at controlling and reducing the risk of this uncertainty that exists in IT2FNs by using FOU.

The quantitative utility (QU) for each alternative should be calculated. The degree of each alternative's utility is directly related to its obtained $H$ value. The degree of an alternative's utility can be computed as

At the end of this process, each alternative gains a score which is presented by $Q U_{i}$. This score demonstrates the desirability of each alternative considering

In this section, a model is presented that is aiming at obtaining a portfolio of projects that suits all the existing criteria of the process in the best possible way. Notations used in this section are described as

$$
\begin{aligned}
& \left(i t 2 f i_{1}^{U}, i t 2 f i_{2}^{U}, i t 2 f_{3}^{U}, i t 2 f_{4}^{U} ; H_{1}\left(i t 2 f i_{1}^{U}\right),\right. \\
& \left.H_{2}\left(i t 2 f i_{1}^{U}\right)\right), \\
& \left(i t 2 f i_{1}^{L}, i t 2 f i_{2}^{L}, i t 2 f i_{3}^{L}, i t 2 f i_{4}^{L} ; H_{1}\left(i t 2 f i_{1}^{L}\right),\right. \\
& \left.H_{2}\left(i t 2 f i_{1}^{L}\right)\right),
\end{aligned}
$$

$\operatorname{Min}_{I}$, minimum amount if acceptable investment,

$\operatorname{Max}_{I}$, maximum amount of acceptable invest-
IT2F human resource requirement of project $i$

$\operatorname{Max}_{H R}$, maximum level of available human resource,

$x_{i}$, decision variable which is defined by:

$x_{i}=\left\{\begin{array}{l}0 \text { if project } i \text { is rejected } \\ 1 \text { if project } i \text { is selected }\end{array}\right.$

$Z_{2}=\max \sum_{i=1}^{m} x_{i} Q U_{i}$

Subject to :

$\left(\begin{array}{c}i t 2 f i_{1}^{U}, i t 2 f i_{2}^{U}, i t 2 f_{3}^{U}, i t 2 f_{4}^{U} ; \\ H_{1}\left(i t 2 f i_{1}^{U}\right), H_{2}\left(i t 2 f i_{1}^{U}\right)\end{array}\right)$,

$\operatorname{Min}_{I} \leq \sum_{i=1}^{n}\left(\begin{array}{c}i t 2 f i_{1}^{L}, i t 2 f i_{2}^{L}, i t 2 f i_{3}^{L}, i t 2 f i_{4}^{L} ; \\ H_{1}\left(i t 2 f i_{1}^{L}\right), H_{2}\left(i t 2 f i_{1}^{L}\right)\end{array}\right) \leq \operatorname{Max}_{I}$

$$
\begin{aligned}
& \sum_{n}^{n}\left(\begin{array}{c}
I T 2 F H R i_{1}^{U}, I T 2 F H R i_{2}^{U}, I T 2 F H R i_{3}^{U}, \\
I T 2 F H R i_{4}^{U} ; H_{1}\left(I T 2 F H R i_{1}^{U}\right), H_{2}\left(I T 2 F H R i_{1}^{U}\right)
\end{array}\right), \\
& \sum_{i=1}\left(\begin{array}{c}
I T 2 F H R i_{1}^{L}, I T 2 F H R i_{2}^{L}, I T 2 F H R i_{3}^{L}, \\
I T 2 F H R i_{4}^{L} ; H_{1}\left(I T 2 F H R i_{1}^{L}\right), H_{2}\left(I T 2 F H R i_{1}^{L}\right)
\end{array}\right) \cdot x_{i} \\
& \leq \operatorname{Max}_{H R}
\end{aligned}
$$

$$
\left(\begin{array}{l}
i t 2 f i_{1}^{U}, i t 2 f i_{2}^{U}, i t 2 f i_{3}^{U}, \\
i t 2 f i_{4}^{U} ; H_{1}\left(i t 2 f i_{1}^{U}\right), H_{2}\left(i t 2 f i_{1}^{U}\right)
\end{array}\right),
$$$$
\sum_{i \in \text { short-term }}\left(\begin{array}{c}
i t 2 f i_{1}^{L}, i t 2 f i_{2}^{L}, i t 2 f i_{3}^{L}, i t 2 f i_{4}^{L} ; \\
H_{1}\left(i t 2 f i_{1}^{L}\right), H_{2}\left(i t 2 f i_{1}^{L}\right)
\end{array}\right) \cdot x_{i}
$$$$
\leq \frac{\alpha}{\mu} \sum_{i=1}^{N}\left(\begin{array}{c}
i t 2 f i_{1}^{U}, i t 2 f i_{2}^{U}, i t 2 f i_{3}^{U}, i t 2 f i_{4}^{U} ; \\
H_{1}\left(i t 2 f i_{1}^{U}\right), H_{2}\left(i t 2 f i_{1}^{U}\right)
\end{array}\right),
$$$$
\sum_{i \in \text { mid-term }}\left(\begin{array}{c}
i t 2 f i_{1}^{U}, i t 2 f i_{2}^{U}, i t 2 f i_{3}^{U}, i t 2 f i_{4}^{U} ; \\
H_{1}\left(i t 2 f i_{1}^{U}\right), H_{2}\left(i t 2 f i_{1}^{U}\right)
\end{array}\right),
$$ 


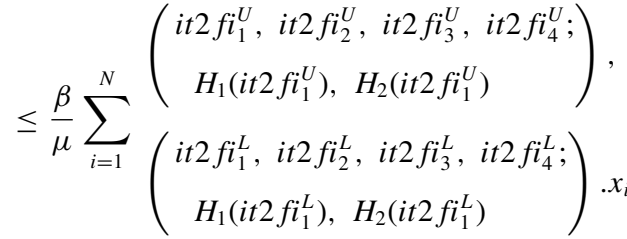

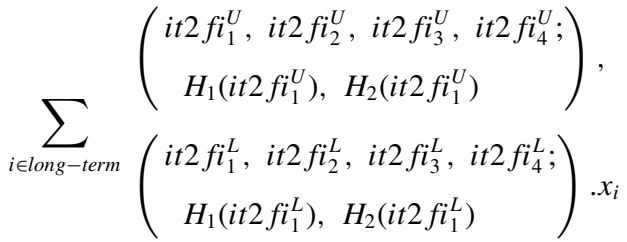

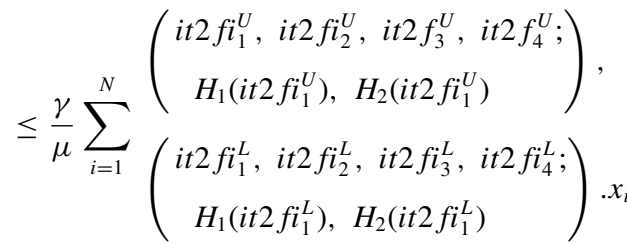

$\alpha+\beta+\gamma=\mu$

$x_{i} \neq x_{i}^{\prime \prime} \quad$ for $i=1,2, \ldots, n ;\left(i, i^{\prime \prime}\right) \in K$

$x_{i}=1 \quad$ fori $=1,2, \ldots, n ; \forall i \in L$

Equation (37) keeps the amount of investment in the feasible region. Equation (38) keeps the number of human resource of the entire selected portfolio in the practical area. Equations (39-41) can be added to the model to plan short, mid and long-term time horizons. Equation (43) indicates the mutual exclusiveness relationship of projects. Equation (44) makes inclusion of a certain project in the portfolio compulsory.

To solve the mathematical model with IT2FSs embedded in the constraints, the concept of expected value defined by $\mathrm{Hu}$ et al. [6] was used. In this approach, each IT2FN used in the model is transformed to crisp value. The following presents the applied approach of transformation:

$$
\begin{aligned}
E(A)= & \frac{1}{2}\left(\frac{1}{3} \sum_{i=1}^{3} a_{i}^{L}+a_{i}^{U}\right) \\
& \times \frac{1}{4}\left(\sum_{i=1}^{2}\left(H_{i}\left(A^{L}\right)+H_{i}\left(A^{U}\right)\right)\right)
\end{aligned}
$$

\subsection{Procedure of the proposed project portfolio selection approach}

In sum, the algorithm is provided by means of the following steps:
Step 1. Provide individual decision information for each DM. Each DM expresses his/her decision matrix. Their decision matrixes are gathered as expressed in Equations (5 and 6).

Step 2. Normalize the gathered decision matrixes by Equations (8 and 9).

Step 3. Construct the weighted (on attributes) individual decision by Equation (13).

Step 4. Determine the ideal decisions of all individual decisions. The best decision $\left(G^{*}\right)$, the left negative ideal decision $\left(G_{l}^{-}\right)$and the right negative ideal decision $\left(G_{r}^{-}\right)$are calculated by Equations (14-16), respectively.

Step 5. Compute the separations of each individual judgment from the best judgment $\left(G^{*}\right)$, the left negative ideal decision $\left(G_{l}^{-}\right)$and the right negative ideal decision $\left(G_{r}^{-}\right)$applying Equations (17-19), respectively.

Step 6. Decide the closeness coefficient of each individual judgment to supreme judgments by using Equation (20).

Step 7. Find the comprehensive closeness coefficient of each DM by employing Equation (21).

Step 8. Obtain the weights of DMs by using Equation (22).

Step 9. Create a decision matrix that is weighted on attributes and DMs for each DM by Equation (23).

Step 10. Convert the individual decision that is weighted on attributes and DMs into the group decision for each alternative by using Equation (24).

Step 11. Solve the mathematical model presented in Equations (25-34) for each alternative.

Step 12. Calculate the quantitative utility of each alternative by using Equation (35).

Step 13. Form the final objective function of the project portfolio selection model by using the obtained quantitative utility.

Step 14. Gather the data concerning the constraints and the limitations and form the final model.

Step 15. Solve the mathematical model to achieve the optimal portfolio of projects. 


\section{Proposed approach application}

In this part, an existing problem in the recent literature is adopted and solved using the proposed approach. Furthermore, the model is presented in two parts and each part is illustratively dealt with by the model.

\subsection{First part of the proposed model}

In this section, to display model's applicability in real-world problems, the data from the case study of Tavana et al. [16] is applied. The main objective of the studied organization is to find the most suitable projects for funding depending on the annual budget constraints.

The following criteria were considered in the problem: Total cost $\left(C_{1}\right)$, Production time $\left(C_{2}\right)$, System safety $\left(C_{3}\right)$, System reliability $\left(C_{4}\right)$, Feasibility $\left(C_{5}\right)$ and eventually, reusability $\left(C_{6}\right) .5$ projects $\left(P_{1}\right)-$ $\left(P_{5}\right)$ from the studied case are selected to be used in the proposed method. A group consisting of 5 experts have expressed their ideas.

Since the calculations are too large to be fully displayed, partial calculations are presented as follows. The closeness coefficient of the individual judgment with respect to supreme judgments is obtained and displayed in Table $2 . \pi_{k}$ is then calculated. It should be noticed that each DM was given the $I M_{k}$ of 0.2 and $\alpha$ was equal to 0.5. $\pi_{k}$ is also displayed in Table 2 . Finally, the weights of DMs are calculated. They also are displayed in Table 2. The initial judgments are weighted by using Equation (23).

The weighted (on attributes and DMs) decision matrix $(S)$ for each DM is aggregated before being used in the mathematical model. The aggregation is carried by applying the following:

$$
\left(\begin{array}{c}
\frac{\sum_{k=1}^{K} s_{k i j 1}^{L}}{K}, \frac{\sum_{k=1}^{K} s_{k i j 2}^{L}}{K}, \frac{\sum_{k=1}^{K} s_{k i j 3}^{L}}{K} \frac{\sum_{k=1}^{K} s_{k i j 4}^{L}}{K} ; \\
\min H_{1}\left(\tilde{S}_{k i j}^{L}\right), H_{2}\left(\tilde{S}_{k i j}^{L}\right), \\
\frac{\sum_{k=1}^{K} s_{k i j 1}^{L}}{K}, \frac{\sum_{k=1}^{K} s_{k i j 2}^{L}}{K}, \frac{\sum_{k=1}^{K} s_{k i j 3}^{L}}{K} \frac{\sum_{k=1}^{K} s_{k i j 4}^{L}}{K} ; \\
\min H_{1}\left(\tilde{S}_{k i j}^{L}\right), H_{2}\left(\tilde{S}_{k i j}^{L}\right),
\end{array}\right)
$$

It should be mentioned that the aforementioned steps are carried out for all the gathered judgments. Eventually, the mathematical model for each alternative is solved. It should be noted that maximum level of uncertainty is set equal to 0.5. $H_{i}, Q U_{i}$ and the results of the existing literature are displayed
Table 2

The closeness coefficient, $\pi_{k}$ and $\mu_{k}$

\begin{tabular}{lccc}
\hline Decision Maker & $C C_{k}$ & $\pi_{k}$ & $\mu_{k}$ \\
\hline$D M_{1}$ & 0.65 & 0.42 & 0.18 \\
$D M_{2}$ & 0.83 & 0.51 & 0.22 \\
$D M_{3}$ & 0.67 & 0.43 & 0.18 \\
$D M_{4}$ & 0.82 & 0.51 & 0.22 \\
$D M_{5}$ & 0.67 & 0.43 & 0.18 \\
\hline
\end{tabular}

Table 3

Final computational results

\begin{tabular}{lcccc}
\hline Projects & $H_{i}$ & $Q U_{i}$ & $\begin{array}{c}\text { Proposed } \\
\text { approach } \\
\text { ranking }\end{array}$ & $\begin{array}{c}\text { Tavana } \\
\text { et al. } \\
{[16]}\end{array}$ \\
\hline$P_{1}$ & 7.122591 & 100 & 1 & 1 \\
$P_{2}$ & 6.102921 & 85.684 & 2 & 2 \\
$P_{3}$ & 5.979368 & 83.94935 & 3 & 3 \\
$P_{4}$ & 4.437909 & 62.30751 & 4 & 4 \\
$P_{5}$ & 3.072105 & 43.13185 & 5 & 5 \\
\hline
\end{tabular}

in Table 3. The results show the reliability if the proposed model in addition to its novelty in giving weights to each DM depending on the achieved judgments.

\subsection{The second part of the proposed approach}

Since the provided case study lacked the required data for this part of the model, in order to display application of this part, the problem is adapted and the required data is added. Table 4 displays the adapted data for each project. To demonstrate model's ability to handle problems under different scenarios, different levels of constraints are considered, and the model is solved under those different constraints. Table 5 displays the achieved results.

\subsection{Model's advantages over similar studies}

Applying the proposed model in the existing literature demonstrated several advantages. The most important implications of the model's application are as follows: (1) the model is based on type 2 FSs. This uncertainty-modeling tool gives the model a practical edge over the existing classic fuzzy models; (2) the model is in two main parts, and it means that the DM can easily observe the results of judgments on projects before choosing the portfolio. Furthermore, uncertain data concerning both quantifiable and unquantifiable can be applied in each part of the model; (3) each DM is given a weight that is based on the expertise and importance of the expert in any studying field, in addition to the data gathered from 
Table 4

Adapted data of the studied projects

\begin{tabular}{lll}
\hline Projects & IT2F investment (million dollars) & IT2F human resource (persons) \\
\hline$P_{1}$ & $((160,180,210,230 ; 1,1),(170,190,200,220 ; 0.9,0.9))$ & $((20,30,45,55 ; 1,1),(25,35,40,50 ; 0.9,0.9))$ \\
$P_{2}$ & $((260,280,310,330 ; 1,1),(270,290,300,320 ; 0.9,0.9))$ & $((15,25,40,50 ; 1,1),(20,30,35,45 ; 0.9,0.9))$ \\
$P_{3}$ & $((110,130,160,180 ; 1,1),(120,140,150,170 ; 0.9,0.9))$ & $((0,10,25,35 ; 1,1),(5,15,20,30 ; 0.9,0.9))$ \\
$P_{4}$ & $((60,80,110,130 ; 1,1),(70,90,100,120 ; 0.9,0.9))$ & $((7,12,27,37 ; 1,1),(7,17,22,32 ; 0.9,0.9))$ \\
$P_{5}$ & $((210,230,260,280 ; 1,1),(220,240,250,270 ; 0.9,0.9))$ & $((10,20,35,45 ; 1,1),(15,25,30,40 ; 0.9,0.9))$ \\
\hline
\end{tabular}

Table 5

Results of the second part of the model

\begin{tabular}{lcccc}
\hline Projects & Budget 0-100 & Budget 100-200 & Budget 200-300 & Budget 0-500 \\
\cline { 2 - 5 } & Human resource 0-30 & Human resource 30-50 & Human resource 50-70 & Human resource 0-120 \\
\hline$P_{1}$ & 0 & 1 & 1 & 1 \\
$P_{2}$ & 0 & 0 & 0 & 0 \\
$P_{3}$ & 0 & 0 & 0 & 1 \\
$P_{4}$ & 1 & 0 & 1 & 1 \\
$P_{5}$ & 0 & 0 & 0 & 0 \\
Objective & 62.3 & 100 & 162.3 & 264.2 \\
\hline
\end{tabular}

other experts; (4) the approach avoids information loss in the decision-making process.

\section{Conclusions}

New technology-project selection is one of the most important tasks of many organizations. Since high technology-projects are nowadays very crucial to advancements of science and technology, and they have not been comprehensively addressed in project selection literature, this paper proposed a novel approach of high technology-project selection. Moreover, the presented approach was in two main parts. In the first part, a new multi criteria decision-making model that avoids information loss was presented that was able to review and rank the projects. In the second part, a model of project portfolio selection was presented that simultaneously considered investments requirements and human resource requirements in finding the optimum portfolio of high technologyprojects. To displays the model's application, a case study for the high technology-project selection problem from the existing literature was chosen and adopted properly to be solved by the model. Applying the approach provided several implications that were discussed. Finally, for further researches, integrating the proposed model in decision support systems could be a practical and interesting work.

\section{Acknowledgments}

The authors would like to thank four anonymous referees for their useful comments on this paper.
References

[1] D. Mandic, P. Jovanovic and M. Bugarinovic, Twophase model for multi-criteria project ranking: Serbian Railways case study, Transport Policy 36 (2014), 88-104.

[2] D. Wu and J.M. Mendel, Computing with words for hierarchical decision making applied to evaluating a weapon system, IEEE Transactions on Fuzzy Systems 18(3) (2010), 441-460.

[3] F. Perez and T. Gomez, Multiobjective project portfolio selection with fuzzy constraints, Annals of Operations Research 245(1) (2016), 7-29.

[4] H.B. Mitchell, Pattern recognition using type-II fuzzy sets, Information Sciences 170(2) (2005), 409-418.

[5] H. Deng, Comparing and ranking fuzzy numbers using ideal solutions, Applied Mathematical Modelling 38(5) (2014), 1638-1646.

[6] J. Hu, Y. Zhang, X. Chen and Y. Liu, Multi-criteria decision making method based on possibility degree of interval type-2 fuzzy number, Knowledge-Based Systems 43 (2013), 21-29.

[7] J.M. Mendel, R. John and F. Liu, Interval type-2 fuzzy logic systems made simple, IEEE Transactions on Fuzzy Systems 14(6) (2006), 808-821.

[8] J.M. Mendel, Type-2 fuzzy sets and systems: An overview, Computational Intelligence Magazine, IEEE 2(1) (2007), 20-29.

[9] J. Qin, X. Liu and W. Pedrycz, An extended VIKOR method based on prospect theory for multiple attribute decision making under interval type-2 fuzzy environment, Knowledge-Based Systems 86 (2015), 116-130.

[10] J. Thomas and T. Mengel, Preparing project managers to deal with complexity-Advanced project management education, International Journal of Project Management 26(3) (2008), 304-315.

[11] J. Wang, Y. Xu and Z. Li, Research on project selection system of pre-evaluation of engineering design project bidding, International Journal of Project Management 27(6) (2009), 584-599.

[12] J.M. Mendel, Type-2 fuzzy sets: Some questions and answers, IEEE Connections, Newsletter of the IEEE Neural Networks Society 1 (2003), 10-13. 
[13] L.A. Zadeh, The concept of a linguistic variable and its application to approximate reasoning, Springer US, 1974, pp. 1-10.

[14] L. Ren, Y. Zhang, Y. Wang and Z. Sun, Comparative analysis of a novel M-TOPSIS method and TOPSIS, Applied Mathematics Research Express (2007). DOI: 10.1093/amrx/abm005

[15] M. Kiliç and İ. Kaya, Investment project evaluation by a decision making methodology based on type-2 fuzzy sets, Applied Soft Computing 27 (2015), 399-410.

[16] M. Tavana, K. Khalili-Damghani and S. Sadi-Nezhad, A fuzzy group data envelopment analysis model for hightechnology project selection: A case study at NASA, Computers \& Industrial Engineering 66(1) (2013), 10-23.

[17] P. Crosby, Characteristics and techniques of successful high-technology project managers, International Journal of Project Organization and Management 4(2) (2012), 99-122.

[18] P. Crosby, Success in large high-technology projects: What really works? In SPIE Astronomical Telescopes + Instrumentation International Society for Optics and Photonics, 2014, pp. 915002-915002.

[19] P. Kundu, S. Kar and M. Maiti, A fuzzy multi-criteria group decision making based on ranking interval type-2 fuzzy variables and an application to transportation mode selection problem, Soft Computing (2015), 1-12.

[20] S.M. Chen and L.W. Lee, Fuzzy multiple attributes group decision-making based on the ranking values and the arithmetic operations of interval type- 2 fuzzy sets, Expert Systems with Applications 37(1) (2010), 824-833.

[21] S. Ebrahimnejad, S.M. Mousavi, R. Tavakkoli-Moghaddam and M. Heydar, Risk ranking in mega projects by fuzzy compromise approach: A comparative analysis, Journal of Intelligent and Fuzzy Systems 26 (2014), 949-959.

[22] S.M. Mousavi, B. Vahdani and M. Abdollahzade, An intelligent model for cost prediction in new product development projects, Journal of Intelligent and Fuzzy Systems 29(5) (2015), 2047-2057.

[23] S.M. Mousavi, B. Vahdani, H. Hashemi and S. Ebrahimnejad, An artificial intelligence model-based locally linear neuro-fuzzy for construction project selection, Journal of Multiple-Valued Logic \& Soft Computing 25(6) (2015), 589-604.

[24] S.M. Mousavi, R. Tavakkoli-Moghaddam, B. Vahdani, H. Hashemi and M.J. Sanjari, A new support vector model-based imperialist competitive algorithm for time estimation in new product development projects, Robotics and Computer-Integrated Manufacturing 29 (2013), 157-168.

[25] T. Dereli and K. Altun, Technology evaluation through the use of interval type-2 fuzzy sets and systems, Computers \& Industrial Engineering 65(4) (2013), 624-633.

[26] T. Dereli and K. Altun, Imovator's Dilemma, How to Decide When to be Offensive and When to be Defensive? In Strategic Planning Decisions in the High Tech Industry, Springer London, 2013, pp. 121-141.

[27] V. Mohagheghi, S.M. Mousavi and B. Vahdani, A new optimization model for project portfolio selection under interval-valued fuzzy environment, Arabian Journal for Science and Engineering 40(11) (2015), 3351-3361.

[28] V. Mohagheghi, S.M. Mousavi and B. Vahdani, Analyzing project cash flow by a new interval type- 2 fuzzy model with an application to construction industry, Neural Computing and Applications (2016). Article in press, DOI: 10.1007/s00521-016-2235-6

[29] V. Mohagheghi, S.M. Mousavi, B. Vahdani and M.R. Shahriari, R\&D project evaluation and project portfolio selection by a new interval type- 2 fuzzy optimization approach, Neural Computing and Applications (2016). Article in press, DOI: 10.1007/s00521-016-2262-3

[30] V. Mohagheghi, S.M. Mousavi and A. Siadat, A new approach in considering vagueness and lack of knowledge for selecting sustainable portfolio of production projects. In Industrial Engineering and Engineering Management (IEEM), 2015 IEEE International Conference on, 2015, pp. 1732-1736. IEEE.

[31] V. Mohagheghi, S.M. Mousavi and B. Vahdani, A new multi-objective optimization approach for sustainable project portfolio selection: A real-world application under interval-valued fuzzy environment, Iranian Journal of Fuzzy Systems 13(6) (2016), 41-68.

[32] Z. Yue, A method for group decision-making based on determining weights of decision makers using TOPSIS, Applied Mathematical Modelling 35(4) (2011), 1926-1936.

[33] Z. Yue, An avoiding information loss approach to group decision making, Applied Mathematical Modelling 37(1) (2013), 112-126.

[34] Z. Zhang and S. Zhang, A novel approach to multi attribute group decision making based on trapezoidal interval type2 fuzzy soft sets, Applied Mathematical Modelling 37(7) (2013), 4948-4971. 